\title{
A importância da avaliação do circuito e gestão da medicação nas instituições de longa permanência para idosos (ILPI): identificação de problemas relacionado com os medicamentos
}

\author{
The importance of circuit evaluation and drug \\ management in residential structures for elderly people: \\ identification of drug related problems
}

Recebido em: 02/04/2019 Aceito em: 02/08/2019
Renato FERREIRA DA SILVA ${ }^{1,2}$; Carina VIEIRA ${ }^{1}$

${ }^{1}$ Faculdade de Farmácia da Universidade do Porto. Rua Jorge de Viterbo Ferreira 228, CP 4050-313. Porto, Portugal; ${ }^{2}$ Porto4Ageing Consortium, Universidade do Porto. Praça de Gomes Teixeira, CP 4050-526. Porto, Portugal.

E-mail: renato.ivos@gmail.com

\section{ABSTRACT}

If evaluating procedures is essential in all areas, in health their relevance has an added role and implies in the creation of systems that allow the evaluation of the medicine circuit and management in spaces where the elder lives, assumes an importance that has to be appropriately framed. It is well known that many Drug Related Problems (DRM) can be easily identified when there are efficient integrated control systems, reducing the risk of exposure to drug errors. Throughout this technical note, we have tried to analyze some published research in this area. Different studies with different protocols evaluated multiple variables in drug circuit and management in Long-Term Care Institutions for the Elderly (LSIE). Thus, from the analysis of these studies, we raised some points that can and should be further developed in the future, with the pharmacist playing an active role in this matter.

KEYWORDS: aging; pharmaceutical care; medication management

\section{RESUMO}

Se avaliar procedimentos é importante em todas as áreas, na saúde a sua relevância tem um papel acrescido. Essa preocupação na criação de sistemas que permitam avaliar o circuito e gestão dos medicamentos em espaços onde residem idosos, assume uma importância que tem de ser devidamente enquadrada. É notório que muitos Problemas Relacionados com os Medicamentos (PRM) podem ser facilmente identificados quando existem sistemas integrados de controlo eficientes, diminuindo o risco de exposição a erros de medicamentos.Ao longo desta nota técnica, procuramos analisar alguns trabalhos publicados nos últimos anos, nest área. Diferentes estudos com diferentes protocolos, avaliaram múltiplas variáveis no circuito e gestão do medicamento em Instituições de Longa Permanência para Idosos (ILPI). Assim, da análise desses estudos, levantamos alguns pontos que podem e devem ser mais aprofundados no futuro, tendo o farmacêutico um papel ativo neste assunto.

PALAVRAS-CHAVE: envelhecimento; cuidados farmacêuticos; gerenciamento da medicação 


\section{INTRODUÇÃO}

O envelhecimento da população é uma realidade demográfica que tem um forte impacto na sociedade. Segundo dados do Censo 2011, mais de 19\% da população portuguesa tinha 65 ou mais anos, o que leva a crer que a institucionalização dos idosos em Portugal é uma realidade muito marcada (1). Apesar dos anos de vida saudáveis após os 65 anos, estes não correspondem necessariamente à classificação do País em termos de longevidade, levantando-se assim uma série de questões para entender as possíveis causas (2). Paralelamente a estes dados, Portugal é também um dos países da Europa onde mais medicamentos são dispensados nas farmácias e onde o seu consumo está intimamente relacionado com uma maior despesa em saúde (dados de 2002, indicam uma relação de 23 embalagens de medicamentos per capita) (3).

Com o aumento da esperança média de vida e do envelhecimento da população, há um aumento da prevalência de variadas patologias, nomeadamente as doenças crônicas e incapacitantes, levando a situações de maior dependência por parte dos idosos (1). Embora os medicamentos estejam fortemente associados a uma melhoria da qualidade de vida da população, a faixa etária aqui em discussão, sobretudo pela razão de apresentar mais comorbidades decorrentes do processo natural de envelhecimento, contribui com uma maior despesa em cuidados de saúde, cuidados assistenciais, medicação e dispositivos médicos (1).

Existem várias formas de definir "polimedicação", podendo ser caracterizada tendo em conta a duração do tratamento e o número de medicamentos tomados. Sendo assim, há "polimedicação" quando um indivíduo toma cinco ou mais medicamentos a nível domiciliar, ou então dez ou mais a nível de internamento. Do ponto de vista da duração do tratamento, um indivíduo é considerado polimedicado quando toma dois ou mais medicamentos por mais de 240 dias ou então cinco a nove medicamentos por 90 dias ou mais (4).

A existência de várias patologias em simultâneo, a polimedicação, a aplicação de regimes terapêuticos complexos, assim como a existência de problemas associados à farmacodinâmica e à farmacocinética dos diferentes medicamentos no organismo (3), são fatores que estão fortemente associados a um aumento dos Problemas Relacionados com os Medicamentos (PRM). Quanto maior o número de medicamentos utilizados pelo idoso, maior é o risco de interações (5).

No contexto europeu, Portugal é um dos países que mais recorre aos medicamentos. Cerca de $20 \%$ das visitas à urgência dizem respeito à existência de PRM, resultando assim num número elevado de hospitalizações que poderiam ser evitadas ou desnecessárias (3). A Pharmaceutical Care Network Europe (PCNE) postula que um PRM diz respeito a um "evento ou circunstância que envolve a terapêutica farmacológica e que interfere com os resultados desejados na saúde do utente" $(3,5)$.

Um estudo realizado em Portugal entre novembro e dezembro de 2013 , mostrou que $46,8 \%$ dos usuários idosos polimedicados apresentava algum PRM (3). Este estudo descritivo observacional, envolveu seis lares portugueses. Para cada lar foram destacados dois farmacêuticos, sendo que um deles realizava a caracterização da instituição e o outro realizava a revisão da terapêutica dos usuários. Cada lar referenciava cinco ou seis pacientes de acordo com critérios previamente estabelecidos, como por exemplo, ter mais de 65 anos, tomar cinco ou mais medicamentos e ter capacidade para responder perante uma entrevista. Os dados demográficos e clínicos, tais como as comorbidades e medicamentos utilizados, foram obtidos dos registos clínicos do lar. Paralelamente, durante as entrevistas foram recolhidas outras informações clínicas relevantes. Os medicamentos foram classificados tendo por base a Anatomical Therapeutic Chemical Classification e os problemas de saúde foram analisados de acordo com a International Statistical Classification of Diseases and Related Health Problems. Para a identificação de medicamento apropriado ou não apropriado, foram utilizados os critérios STOPP e START e os PRM foram organizados de acordo com a PCNE Classification $(3,6,7)$.

Nenhum dos lares tinha o apoio de um farmacêutico e a avaliação dos parâmetros bioquímicos e fisiológicos não era realizada por profissionais de saúde. A amostra em estudo incluiu 31 idosos, na 
maioria do sexo feminino $(64,52 \%)$. Os idosos deste lar apresentavam em média 7,94 problemas de saúde. Dentre estes, os mais usuais eram as doenças do sistema circulatório, seguidos pelos problemas do foro endócrino, nutricional e metabólico e, por último, os problemas digestivos e do Sistema Nervoso Central (SNC) (3).

No estudo foram identificados 484 PRM, calculando-se uma média de 15 PRM por idoso. Desta forma, 63,45\% eram potenciais PRM e 36,45 eram PRM manifestos. O PRM (potencial e manifesto) mais frequente, dizia respeito a reações adversas ao medicamento e a possíveis interações medicamentosas. Por outro lado, o PRM mais manifestado estava relacionado com o tratamento farmacológico mais dispendioso que o necessário $(3,5,8)$. Para os dois PRM anteriores, a causa residia na combinação inapropriada de medicamentos e/ou alimentos, bem como utilização de fármacos com maior custo/ efetividade. Para além disto, o efeito do fármaco não ser o ótimo, ou ser desnecessário para o tratamento, e a administração não ser feita no tempo certo, também eram outras das causas apontadas pelos investigadores $(3,5,8)$.

As prescrições inapropriadas ou a omissão de outros medicamentos podem ser avaliadas por meio dos critérios START e STOPP. Os critérios START estão relacionados com os medicamentos que são necessários para melhorar o estado de saúde de um indivíduo, mas que foram omitidos da sua terapêutica. Pelo contrário, os critérios STOPP dizem respeito a medicamentos que estão associados a efeitos adversos. Estes últimos referem-se a sessenta e cinco pontos, divididos por cinco subsistemas fisiológicos, de forma a tornar mais simples a análise da medicação do doente. O mesmo acontece com os critérios START, que correspondem a vinte e dois critérios também divididos por categorias respeitantes a seis sistemas fisiológicos. Estes critérios pretendem definir a mais valia de determinado fármaco em determinada terapêutica $(9,10)$.

Por meio dos critérios START e STOPP, foi possível identificar 69 indicações de medicamentos potencialmente inapropriados (STOPP) ou potencialmente necessários (START). Assim, do conjunto de problemas identificados, 76,82\% diziam respeito a critérios STOPP, devido ao uso a longo prazo de fármacos que atuam no $\mathrm{SNC}$, de fármacos psicotrópicos e de inibidores da bomba de protões. Por outro lado, relativamente aos critérios START, é possível dizer que $25 \%$ dos idosos necessitava de tomar um inibidor da enzima conversora da angiotensina e $18,75 \%$ necessitava de um suplemento de cálcio e vitamina D (5).

A presença de um farmacêutico nas Instituições de Longa Permanência para Idosos (ILPI), poderia contribuir muito na resolução dos PRM encontrados, tal como, na otimização da terapêutica, bem como na minimização de efeitos secundários associados à mesma $(3,8)$.

Um outro estudo iniciado em 2013 em Portugal, utilizou uma amostra de 126 doentes polimedicados, com idades superiores ou iguais a 65 anos, de 3 lares portugueses para idosos (2). Desta amostra inicial, foi retirada uma amostra para ser submetida à intervenção, no sentido de comparar os resultados com os demais pacientes. Recorrendo a dados clínicos (diagnósticos médicos, exames laboratoriais, biomarcadores clínicos e eventuais hospitalizações), assim como os respectivos medicamentos dos doentes, foram identificados os PRM, tendo por base, para a sua qualificação, a II classificação de Granada: necessidade, efetividade e segurança $(11,12)$. Após esta fase inicial, foram identificados os PRM clinicamente mais significativos, no sentido de serem alvo de intervenções por carta dirigida ao médico prescritor e ao enfermeiro.

Os resultados do estudo dos 126 pacientes, totalizou 1332 medicamentos prescritos, com uma mediana de 14,5 PRM por paciente, o que mostra um forte potencial de intervenção do farmacêutico clínico. No final, foram enviadas 62 cartas com um total de 539 recomendações, sendo que a equipe de investigação só recebeu 172 respostas face às recomendações enviadas inicialmente. Das 172 recomendações respondidas, apenas 15 foram aceites (2). Desta forma, o estudo vem realçar a importância de uma maior colaboração entre os profissionais de saúde, no sentido de aumentar a aceitabilidade das recomendações elaboradas pelo farmacêutico clínico.

Um outro trabalho diz respeito a um estudo sueco publicado em 2016, que mostrou a influência do farmacêutico clínico na qualidade das prescri- 
ções médicas (13). Por meio do modelo "Lund Integrated Medicines Management" (LIMM), a equipe de investigação avaliou a qualidade do serviço de farmácia clínica nos cuidados de saúde primários. Neste estudo, foram incluídos todos os idosos com idade igual ou superior a 65 anos, que habitavam em residências comunitárias ou em ILPI. Por meio de um inquérito de 20 questões, o médico de clínica geral, o farmacêutico clínico e o enfermeiro, avaliaram durante duas semanas a sintomatologia associada à terapêutica farmacológica. Os resultados dos inquéritos foram cruzados com outras informações, tais como a pressão arterial, acidentes por quedas, episódios de confusão, assim como com a lista dos medicamentos dos utentes. Neste processo, foram identificados os PRMs, resultando em recomendações ao usuário como resultado de uma discussão com todos os profissionais de saúde da equipe (Quadro 1).

Quadro 1. Principais recomendações dirigidas aos usuários após a identificação dos Problemas Relacionados a Medicamentos (PRM) (13)

\section{RECOMENDAÇÕES AO USUÁRIO}

1. Necessidade de terapêutica adicional.

2. Terapia desnecessária/indicação pouco clara.

3. Medicamento errado/medicamento inadequado para idosos.

4. Dosagem muito baixa.

5. Dosagem muito alta.

6. Reação Adversa ao Medicamento (RAM).

7. Não conformidade.

Os resultados do estudo mostrarm que $87 \%$ dos PRM identificados resultaram em recomendações pelos farmacêuticos clínicos. Das 349 recomendações, 154 (44\%) corresponderam a terapias desnecessárias e 67 (19\%) corresponderam a dosagens muito altas (13). As conclusões deste protocolo vieram evidenciar uma proporção de recomendações clínicas bastante significativas, mostrando uma concordância moderada a alta entre as recomendações dos médicos e dos farmacêuticos clínicos.

\section{PROPOSIÇÃO}

Tendo em atenção a análise sociocultural das faixas etárias em discussão, é importan- te sublinhar que um dos fatores possivelmente implicados no surgimento de PRM, passa pelo uso incorreto do medicamento ou da falta de adesão à terapêutica. Está ainda descrito na literatura, que a falta de colaboração entre os diferentes profissionais de saúde, nomeadamente o médico de medicina geral e familiar e o farmacêutico, está fortemente relacionada com um aumento da morbidade e da mortalidade da terapêutica aplicada (7). Para contornar alguns destes problemas, além de uma maior intercolaboração entre os diferentes prestadores e profissionais de saúde, é necessário que estes, dentro das suas competências, consigam identificar possíveis fontes de problemas, de forma a garantir o máximo benefício terapêutico no idoso e, consequentemente, prevenir o surgimento de Resultados Negativos associados aos Medicamentos (RNM).

Na Figura 1 está esquematizada a proposta de uma sequência de etapas que deverá ser seguida no momento da avaliação do circuito e gestão do medicamento. $\mathrm{O}$ algoritmo proposto, foi elaborado com base no que é considerado como boas práticas de atuação no momento de avaliar todo o circuito e gestão do medicamento em ILPI e, eventualmente, noutro tipo de instituições.

Após a prescrição do médico, o farmacêutico é o responsável pela dispensa dos medicamentos no contexto de farmácia oficinal ou hospitalar. A partir deste ponto, todos os outros dizem respeito à atuação de outros profissionais das ILPI, sendo o farmacêutico um elemento fundamental na formação e aquisição de competências destes profissionais. Uma vez nas ILPI, os medicamentos devem seguir uma cadeia de qualidade e segurança, que deve ser da responsabilidade dos profissionais das ILPI, mas monitorizado periodicamente por um farmacêutico. Com exceção da primeira etapa do algoritmo, as restantes etapas referem-se aos diferentes momentos do circuito do medicamento em que o farmacêutico tem a responsabilidade de atuar.

Em 2018, foi publicado um trabalho de investigação que se traduzia num algoritmo - o $A M B E R$ Study Protocol (14), de forma a desenvolver uma ferramenta de apoio à revisão dos medicamentos em ILPI, como suporte à prática farmacêutica. 


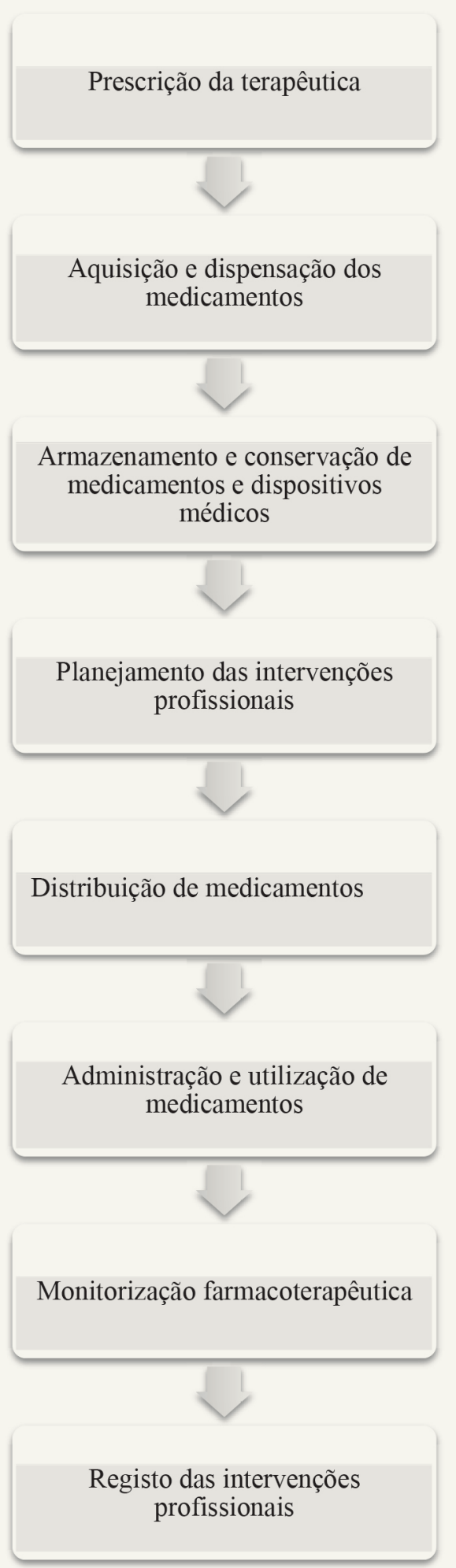

Figura 1. Proposta de algoritmo de avaliação do circuito e gestão dos medicamentos em instituições de longa permanência para idosos (ILPI)
Este algoritmo é constituído por 3 fases: a fase I, onde são realizadas entrevistas aos diversos profissionais de saúde e pacientes, relativamente à sua experiência, exigências e expetativas sobre problemas, riscos e objetivos da utilização dos medicamentos; a fase II, onde é elaborada uma revisão sistemática sobre revisão dos medicamentos em ILPI, combinando os resultados da pesquisa, com a informação extraída das entrevistas da fase I; e a fase III, o algoritmo desenvolvido será testado quando à eficácia, por meio da sua implementação em ILPI no ato de revisão dos medicamentos, sendo que a aceitação das recomendações farmacêuticas será quantificada por meio de formulário próprio (14, $15,16)$.

Apesar dos resultados da implementação deste algoritmo ainda não terem sido publicados, parece evidente que a fase das entrevistas, associada a uma fase posterior de revisão bibliográfica, poderá ser uma mais valia na elaboração de protocolos de revisão dos medicamentos, adaptados às características de cada instituição.

Um outro fator frequentemente não referido quando esta temática é abordada, mas que pode ter um peso relevante quando da avaliação do circuito e gestão dos medicamentos, diz respeito à interrupção do ato de administração de um medicamento. Um estudo publicado em 2018, avaliou a administração do medicamento em ILPI, caracterizando as interrupções do ato de administrar o medicamento ao paciente (17). A complexidade do ato de administração, associado aos múltiplos fatores do meio ambiente, conduzem a erros que muitas vezes não chegam a ser detetados. Para caracterizar o tipo de interrupções, foram criadas três categorias: interrupções passivas (alarmes e ruídos de fundo); interrupções ativas (discussões); e interrupções tecnológicas (dispositivos móveis). Os resultados evidenciaram que a complexidade observada na administração de medicamentos se devia à elevada quantidade de tarefas individuais do pessoal técnico das ILPI, falta de documentação, aparente liberdade da equipa sobre a gestão e o circuito da medicação, entre outros fatores mencionados nos resultados deste estudo. O que é certo, é que além destas situações, foram verificadas altas taxas de interrupções, na sua maioria com resultados negativos para o ato de administração da medicação, 
embora situações pontuais pudessem ter desfechos positivos (advertências em situações de erro) (17).

Os idosos constituem uma fração da população mais vulnerável, seja pelos múltiplos diagnósticos de patologias decorrentes do envelhecimento, ou mesmo pelo aumento do consumo de medicamentos, estando assim mais suscetíveis a PRM, como resultado das alterações fisiológicas do envelhecimento. A evidência da morbimortalidade resultante do consumo de medicamentos na população idosa institucionalizada, traduz-se numa preocupação acrescida, pelo que a discussão aqui apresentada constitui uma forma de alertar para algumas das falhas associadas ao circuito e gestão do medicamento nas ILPI (1). A revisão dos medicamentos, assim como todo o acompanhamento do circuito do medicamento, deve ser liderada pelo farmacêutico, uma vez que corresponde a uma área da responsabilidade da assistência farmacêutica (2). Apesar do farmacêutico estar inserido em equipes multiprofissionais, este deverá assumir funções clínicas diferenciadas dos restantes profissionais de saúde, de forma a garantir uma redução dos erros, intera- ções e PRM associados às terapêuticas instituídas aos pacientes, contribuindo para o uso racional do medicamento.

\section{CONCLUSÃO}

Uma das grandes dificuldades em se conseguir comparar os diferentes estudos sobre PRM utilizados a nível internacional, resulta da utilização de diferentes metodologias e protocolos, por parte das equipes de investigação. Além disso, a maioria dos estudos realizados em Portugal, não utiliza a II classificação de Granada para qualificar os problemas com os medicamentos, apesar de ser a única validada neste País.

Em suma, o farmacêutico tem um papel importante junto deste tipo de instituições geriátricas, no sentido de garantir a correta gestão dos medicamentos, seja do ponto de vista da dispensação dos medicamentos às ILPI, seja no armazenamento ou monitoramento de uso. A identificação de PRM ao longo de todo o processo é fundamental para impedir o surgimento de RNM.

\section{REFERÊNCIAS}

1. PORTUGAL. Instituto Português da Qualidade. Recomendação da Comissão Setorial para a Saúde do Sistema Português da Qualidade - CS/09, de 11 de dezembro de 2014. Gestão da Medicação nas Estruturas Residenciais para Pessoas Idosas (ERPI). Qualidade CSpaSdSPd, editor. 2014.

2. Costa FA, Silvestre L, Periquito C, Carneiro C, Oliveira P, Fernandes AI, et al. Drug-Related Problems Identified in a Sample of Portuguese Institutionalised Elderly Patients and Pharmacists' Interventions to Improve Safety and Effectiveness of Medicines. Drugs Real World Outcomes. 2016;3(1):89-97. DOI: 10.1007/s40801-0160061-x.

3. Silva C, Ramalho C, Luz I, Monteiro J, Fresco P. Drug-related problems in institutionalized, polymedicated elderly patients: opportunities for pharmacist intervention. Int J Clin Pharm. 2015;37(2):327-334. DOI: 10.1007/ s11096-014-0063-2.

4. Masnoon N, Shakib S, Kalisch-Ellett L, Caughey GE. What is polypharmacy? A systematic review of definitions.BMC Geriatr. 2017;17(1):230. DOI: 10.1186/ s12877-017-0621-2.
5. Turnheim K. Drug therapy in the elderly. Exp Gerontol. 2004;39(11-12):1731-8. DOI: 10.1016/j.exger.2004.05.011.

6. Campos AC. Health Reforms - the conductor wire. Edições Almedina. 2008.

7. Guerreiro MP, Cnatrill JM, Pisco L, Martins AP, Considerations on preventable drug related morbidity in primary care part I - impact of preventable drug-related morbidity. Rev Port Clin Geral. 2005:21:269-279.

8. Hazen ACM, Zwart DLM, Poldervaart JM, de Gier JJ, de Wit NJ, de Bont AA, Bouvy ML. Non-dispensing pharmacists' actions and solutions of drug therapy problems among elderly polypharmacy patients in primary care. Fam Pract. 2019. DOI: 10.1093/fampra/cmy114.

9. O’Mahony D, O'Sullivan D, Byrne S, O'Connor MN, Ryan C, Gallagher P. STOPP/START criteria for potentially inappropriate prescribing in older people: version 2 [published correction appears in Age Ageing. 2018 May 1;47(3):489]. Age Ageing. 2015;44(2):213-218. DOI: 10.1093/ageing/afu145. 
10. Periquito CM, Silva PC, Oliveira P, Carneiro C, Fernandes AI, Costa FA. Revisão da medicação em idosos institucionalizados: aplicação dos critérios STOPP e START. Rev Port Farmacoter. 2014;6:211-217. DOI: 10/25756/ rpf.v6i4.20.

11. Committee Consensus. Segundo Consenso de Granada sobre Problemas Relacionados con Medicamentos. Ars Pharm. 2002;43(3-4):175-184.

12. Santos H, Iglesias P, Fernandez-Llimos F, Faus MJ, Rodrigues LM. Segundo Consenso de Granada sobre Problemas Relacionados com Medicamentos. Traducão intercultural de Espanhol para Português (europeu). Acta Med Port. 2004; 17:59-66.

13. Modig S, Holmdahl L, Bondesson A. Medication reviews in primary care in Sweden: importance of clinical pharmacists' recommendations on drug-related problems. Int J Clin Pharm. 2016;38(1):41-45. DOI: 10.1007/s11096015-0189-x.
14. Erzkamp S, Rose O. Development and evaluation of an algorithm-based tool for Medication Management in nursing homes: the AMBER study protocol. BMJ Open 2018;8: e019398. DOI: 10.1136/ bmjopen-2017-019398.

15. Meyboom, Ronald HB, Marie Lindquist, and Antoine CG Egberts. An ABC of drug-related problems. Drug Safety. 2000;22(6):415-423. DOI: 10.2165/00002018200022060-00001.

16. van Mil JW, Westerlund LO, Hersberger KE, Schaefer MA. Drug-related problem classification systems. Ann Pharmacother. 2004;38(5):859-867. DOI: 10.1345/ aph.1D182.

17. Odberg KR, Hansen BS, Aase K, Wangensteen S. Medication administration and interruptions in nursing homes: A qualitative observational study. J Clin Nursing. 2018;27(5-6):1113-1124. DOI: 10.1111/jocn. 14138. 